\title{
Germline knockdown of spargel (PGC-1) produces embryonic lethality in Drosophila
}

\author{
Jack George, Howard T. Jacobs* \\ Faculty of Medicine and Health Technology, Tampere University, Finland
}

\section{A R T I C L E I N F O}

\section{Keywords:}

Mitochondrial biogenesis

Embryogenesis

Transcriptional coactivator

Oogenesis

Drosophila

PGC-1 $\alpha$

\begin{abstract}
A B S T R A C T
The PGC-1 transcriptional coactivators have been proposed as master regulators of mitochondrial biogenesis and energy metabolism. Here we show that the single member of the family in Drosophila, spargel (srl) has an essential role in early development. Female germline-specific RNAi knockdown resulted in embryonic semilethality. Embryos were small, with most suffering a catastrophic derangement of cellularization and gastrulation, although genes dependent on localized determinants were expressed normally. The abundance of mtDNA, representative mitochondrial proteins and mRNAs were not decreased in knockdown ovaries or embryos, indicating that $s r l$ has a more general role in early development than specifically promoting mitochondrial biogenesis.
\end{abstract}

\section{Introduction}

The transcriptional coactivators of the PGC-1 family have been proposed as global regulators of mitochondrial biogenesis and metabolism (Spiegelman, 2007). Three members of the family, PGC-1 $\alpha$, PGC-1 $\beta$ and PPRC1 (or PRC) are encoded in mammalian genomes and are differentially expressed in tissue-specific or physiologically responsive patterns (Finck and Kelly, 2006). For example, PGC-1 $\alpha$ is strongly induced in brown fat when hormonally stimulated (Puigserver and Spiegelman, 2003), whilst PPRC1 is instead induced by the addition of serum to quiescent cells, and has been shown to activate genes connected with the metabolic needs of growing cells (Andersson and Scarpulla, 2001).

In contrast, only a single member of the gene family, spargel ( $(\mathrm{srl})$, is found in Drosophila, facilitating its study without the confounding factors of gene diversification and redundancy. In principle, given the molecular genetic toolkit available in Drosophila, this should allow the metabolic and developmental context of its expression to be elucidated, and its downstream effects on gene expression to be profiled, potentially revealing its ancestral function(s). In one such study, a spontaneously isolated hypomorph of $s r l$ was found to have a metabolic deficiency (in males), comprising decreased weight, and decreased accumulation of storage nutrients (Tiefenböck et al., 2010). Females of the line were sterile, and the mutant phenotypes seen in both sexes were compensated by the transgenic introduction of an additional genomic copy of the wild-type srl gene. Furthermore, alterations to the global pattern of gene expression in the fat body of the mutant males was consistent with a requirement for a sufficient expression of $s r l$ to maintain mitochondrial functions, although many other targets were identified, suggesting that $s r l$ may have a wider function. Although the question of its specificity remains unresolved, subsequent authors have also reported positive effects of $\mathrm{srl}$ on mitochondria and metabolism (Mukherjee et al., 2014), reinforcing the mammalian paradigm of PGC1 function.

The female sterility of the $\operatorname{srl}^{1}$ hypomorph, which is associated with an apparent delay in oogenesis (Mukherjee et al., 2014), could be interpreted in accordance with this paradigm, given that mitochondria and mitochondrial DNA are important components of the oocyte cytoplasm in Drosophila, as in other metazoans. Following their proliferation during oogenesis and delivery into the oocyte (Tourmente et al., 1990), mitochondria are partitioned to the cells of the embryo during cellularization and contribute vital metabolic functions that are tightly regulated during embryogenesis (Akiyama and Okada, 1992) and are essential for the growth of the resulting larva (Galloni, 2003; Adán et al., 2008). As such, a failure of transcription of genes for mitochondrial products during oogenesis would be a logical consequence of $s r l$ downregulation, leading to their catastrophic under-representation in the embryo and larva. Without functional mitochondria, a viable oocyte might fail to form.

In order to test these ideas, and gain insight into the role of $\mathrm{srl}$ in early development, we employed an RNAi approach. Initially we set out to test whether the female sterility caused by $s r l$ downregulation was a

\footnotetext{
* Corresponding author at: Faculty of Medicine and Health Technology, FI-33014, Tampere University, Finland.

E-mail address: howard.jacobs@tuni.fi (H.T. Jacobs).
} 
somatic or germline effect, and whether it affected egg production and/ or viability. To this end, we took advantage of the germline-specific MTD-GAL4 driver (Rørth, 1998; Hudson and Cooley, 2014). By combining this with an RNAi targeted on srl, using the VALIUM20 system (Ni et al., 2011), we confirmed that that the effect was germline-specific. Whilst ovaries appeared normal in germline srl knockdown females, most embryos did not develop properly, with failure to form a uniform blastoderm and gross morphological abnormalities observed from around the time of cellularization onwards. We proceeded to investigate various parameters in an attempt to establish whether $\mathrm{srl}$ knockdown led to a specific defect in the expression of mitochondrial genes or functions, as predicted by the widely held assumption that the PGC-1 coactivators act as master regulators of mitochondrial biogenesis in different contexts. However, the findings suggest an alternative or more global role of $\mathrm{srl}$ in development. Given the reasons for adopting Drosophila as the model organism for this study, our findings suggest that a global role of this kind, lined to growth and transcriptional switching in early development, may be the ancestral function of the PGC-1 coactivators. More specific roles in regard to metabolic reprogramming, energy homeostasis and mitochondrial biogenesis may have evolved subsequently or in parallel.

\section{Materials and methods}

\subsection{Drosophila strains and maintenance}

Oregon R (wild-type) and balancer lines were originally sourced from stock centres. Germline-specific drivers MTD-GAL4 (Bloomington 31777), nos-GAL4 drivers NGT4O (Bloomington 4442) and NGTA (Bloomington 32563) (Tracey Jr et al., 2000; Wheeler et al., 2002), as well as TRiP RNAi lines targeted on spargel: HMS00857 (Bloomington 33914), HMS00858 (Bloomington 33915) and GL01019 (Bloomington 57043), were sourced from Bloomington Stock Center. Flies were maintained at $25^{\circ} \mathrm{C}$ in a $12 \mathrm{~h}$ light-dark cycle in plugged plastic vials containing standard high-sugar medium (Kemppainen et al., 2016), supplemented with $0.5 \%$ propionic acid (Sigma-Aldrich) and $0.1 \%(\mathrm{w} /$ v) methyl 4-hydroxybenzoate (Nipagin, Sigma-Aldrich). For embryo collection, approximately 200 female virgin flies of either Oregon R or one of the respective spargel knockdown lines crossed to MTD- or nosGAL4 drivers were mated with approximately 100 Oregon $\mathrm{R}$ males in mating chambers with standard medium plates and left to lay eggs at $25^{\circ} \mathrm{C}$ for the times indicated in figure legends.

\subsection{Developmental assays}

Batches of 10 female virgin flies of a given genotype were pre-mated with five Oregon $\mathrm{R}$ males on standard medium for 2 days, then tipped to fresh vials and allowed to lay eggs over a $24 \mathrm{~h}$ period. The adult flies were then discarded and the laid eggs were counted. The number of adult flies eventually eclosing from each vial was also counted to generate a percentage based on the number of embryos that completed development, using 5-20 vials for each genotype. To measure fecundity, females of a given genotype were mated as above to Oregon $\mathrm{R}$ males that were then discarded. Females were tipped to fresh vials every 5 days, and the number of eggs laid over a $24 \mathrm{~h}$ period measured four times, i.e. at days 5, 10, 15 and 20.

\section{3. mtDNA copy number measurement}

Batches of 10 ovary pairs or 50-200 embryos were crushed in $500 \mu \mathrm{l}$ DNA lysis buffer $(75 \mathrm{mM} \mathrm{NaCl}, 50 \mathrm{mM}$ EDTA $20 \mathrm{mM}$ HEPES/NaOH, $\mathrm{pH} 7.8) .5 \mu \mathrm{l}$ of $20 \%$ SDS and $20 \mu \mathrm{l}$ of Proteinase $\mathrm{K}(10 \mathrm{mg} / \mathrm{ml}$, ThermoFisher Scientific) were added to each sample and vortexed to mix. Samples were briefly centrifuged, left on a heat block at $50{ }^{\circ} \mathrm{C}$ for $4 \mathrm{~h}$, then vortexed and centrifuged at $16,000 \mathrm{~g}_{\max }$ for $1 \mathrm{~min}$ to pellet debris. Supernatants were decanted and nucleic acid was precipitated with $420 \mu \mathrm{l}$ of isopropanol with repeated inversion and overnight incubation at $-20{ }^{\circ} \mathrm{C}$. Samples were centrifuged at $16,000 g_{\max }$ for $30 \mathrm{~min}$ at $4{ }^{\circ} \mathrm{C}$ to pellet the DNA, which was washed with $500 \mu$ of ice-cold $70 \%$ ethanol. Final pellets were left to air dry for 10 mins, then resuspended in $100 \mu \mathrm{l}$ of TE buffer ( $10 \mathrm{mM}$ Tris/HCl, $1 \mathrm{mM}$ EDTA, $\mathrm{pH}$ 7.8) overnight at $50{ }^{\circ} \mathrm{C}$. DNA concentration was measured by nano-drop spectrophotometry and samples were diluted to $2.5 \mathrm{ng} / \mu \mathrm{l}$. Relative DNA levels of RpL32 (single-copy nDNA) and mt:lrRNA (16S, mtDNA) were determined by qPCR using Applied Biosystems StepOnePlus ${ }^{\mathrm{TM}}$ Real-Time PCR System with Fast SYBR $^{\mathrm{TM}}$ Green Master Mix kit (Applied Biosystems), using as template $2 \mu \mathrm{l}$ of DNA in a $20 \mu \mathrm{l}$ reaction, together with gene-specific primer pairs each at $500 \mathrm{nM}$, as follows (all shown $5^{\prime}$ to $3^{\prime}$ ): RpL32 - TGTGCACCAGGAACTTCTTGAA and AGGCCCAAGATC GTGAAGAA; mt:IrRNA -ACCTGGCTTACACCGGTTTG and GGGTGTA GCCGTTCAAATTT. mtDNA copy number per haploid copy of nDNA was inferred from the cycle-time difference $\left(\Delta \mathrm{C}_{\mathrm{T}}\right)$ of the two test genes, i.e. $2 \exp \Delta \mathrm{C}_{\mathrm{T}}$. SD was calculated from the mtDNA copy number values in a genotype group, and means and SD values were normalized to that of Oregon R for the given tissue or stage (Quiros et al., 2017).

\subsection{RNA extraction and analysis}

Total RNA was extracted from batches of 20 pairs of ovaries (dissected and placed in PBS), or from 100 to 200 embryos (at 80 or 160 min AEL, dechorionated in 50\% household bleach (Domestos) for 2 min and rinsed thee times in PBS) or from batches of 2-day old adult flies using a plastic homogenizing pestle and trizol reagent as previously described (Kemppainen et al., 2016). cDNA was synthesized using the High-capacity cDNA Reverse Transcription Kit (ThermoFisher Scientific) according to manufacturer's instructions. Expression levels were determined by qRT-PCR using Applied Biosystems StepOnePlus ${ }^{\mathrm{TM}}$ Real-Time PCR System with Fast SYBR ${ }^{\mathrm{TM}}$ Green Master Mix kit (Applied Biosystems) using, as template, $2 \mu \mathrm{l}$ of cDNA product diluted 10 -fold, in a $20 \mu \mathrm{l}$ reaction, together gene-specific primer pairs, each at $50 \mathrm{nM}$, as follows (all given $5^{\prime}$ to $3^{\prime}$, gene symbols in the following list following current practice in FlyBase (Thurmond et al., 2019; Chintapalli et al., 2007)): RpL32 (CG7939), TGTGCACCAGGAACTTCTTGAA and AGGC CCAAGATCGTGAAGAA; $s r l$ (CG9809), GGAGGAAGACGTGCCTTCTG and TACATTCGGTGCTGGTGCTT; ND-ACP (CG9160), ACAAGATCGAT CCCAGCAAG and ATGTCGGCAGGTTTAAGCAG: ND-30 (CG12079), AAGGCGGATAAGCCCACT and GCAATAAGCACCTCCAGCTC; $m t: N D 5$ (CG34083), GGGTGAGATGGTTTAGGACTTG and AAGCTACATCCCCA ATTCGAT; SdhA (CG17246), CATGTACGACACGGTCAAGG and CCTT GCCGAACTTCAGACTC; $S d h D$ (CG10219) GTTGCAATGCCGCAAATCT and GCCACCAGGGTGGAGTAG; RFeSP (CG7361), GGGCAAGTCGGTT ACTTTCA and GCAGTAGTAGCCACCCCAGT; UQCR-C2 (CG4169), GAGGAACGCGCCATTGAG and ACGTAGTGCAGCAGGCTCTC; blw (CG3612), GACTGGTAAGACCGCTCTGG and GGCCAAGTACTGCAGA GGAG; COX5A (CG14724), AGGAGTTCGACAAGCGCTAC and ATAGA GGGTGGCCTTTTGGT; COX4 (CG10664), TCTTCGTGTACGATGAGCTG and GGTTGATTTCCAGGTCGATG; $m t: C y t-b$ (CG34090), GAAAATTCC GAGGGATTCAA and AACTGGTCGAGCTCCAATTC; ATPsynF (CG4692), CTACGGCAAAGCCGATGT and CGCTTTGGGAACACGTACT; $m t: l r R N A$ (CR34094), ACCTGGCTTACACCGGTTTG and GGGTGTAGCCGTTCAA ATTT; TFAM (CG4217), AACCGCTGACTCCCTACTTTC and CGACGGT GGTAATCTGGGG; Gapdh1 (CG12055) GACGAAATCAAGGCTAAGG TCG and AATGGGTGTCGCTGAAGAAGTC. Mean values were normalized first against that for RpL32 and then against an arbitrary standard, usually Oregon $\mathrm{R}$ from the same stage or material, as indicated in figure legends. Statistical analysis used the primary $\mathrm{dC}_{\mathrm{T}}$ data, whilst plotted data shows the normalized fold-changes.

\subsection{Protein analysis}

Batches of 20 pairs of ovaries (dissected and placed in PBS) or of 100-200 embryos at 80 or 160 mins AEL, dechorionated in $50 \%$ 
household bleach (Domestos) for $2 \mathrm{~min}$ and rinsed three times in PBS) were crushed in $50 \mu \mathrm{l}$ lysis buffer (0.3\% SDS in PBS, plus one EDTA-free cOmplete $^{\mathrm{TM}}$ Protease Inhibitor Cocktail tablet (Roche) per $10 \mathrm{ml}$ ). After $15 \mathrm{~min}$ incubation at room temperature, samples were centrifuged at $15,000 g_{\max }$ for $10 \mathrm{~min}$ at room temperature. Supernatants were decanted and protein concentrations determined using the Bradford assay. Samples were ran on AnyKD ${ }^{\mathrm{TM}}$ Criterion $^{\mathrm{TM}} \mathrm{TGX}^{\mathrm{TM}}$ Midi gels (Bio-Rad) in ProSieve $^{\mathrm{TM}}$ EX running buffer (Lonza) with either Prestained Pageruler ${ }^{\mathrm{TM}}$ (or Pageruler ${ }^{\mathrm{TM}}$ Plus) Ladder (ThermoFisher Scientific), or Spectra ${ }^{\mathrm{TM}}$ Multicolor Broad Range Protein Ladder (ThermoFisher Scientific) as molecular weight markers. Transfer was performed using the TransBlot $^{\circledR}$ Turbo $^{\mathrm{TM}}$ Transfer System (Bio-Rad) with the midi nitrocellulosespecific transfer pack. Membranes were blocked in 5\% non-fat milk in PBS-0.05\% Tween (Medicago) for $30 \mathrm{~min}$ at room temperature with gentle shaking, then incubated similarly with primary antibodies, as listed below, at $4{ }^{\circ} \mathrm{C}$ overnight. After three 10 min washes in PBS- $0.05 \%$ Tween, membranes were incubated with secondary antibody in the same buffer for $2 \mathrm{~h}$ at room temperature, and washed twice in PBS$0.05 \%$ Tween and then for a final $10 \mathrm{~min}$ in PBS. $5 \mathrm{ml}$ of Luminata ${ }^{\mathrm{TM}}$ Crescendo Western HRP substrate solution (Merck) was added for $5 \mathrm{~min}$ before imaging (Bio-Rad ChemiDoc MP). Densitometry was implemented using ImageJ software. Primary antibodies and dilutions were as follows: ATP5A 1:50,000 Abcam\#14748, COXIV 1:1000 Abcam\#16056, NDUFS3 1:2500, Abcam\#14711, $\beta$-actin - DSHB JLA20-s 1:2500 (all mouse monoclonals), GAPDH 1:5000 Everest Biotech EB06377 (goat polyclonal), Histone H4 (Abcam, \#10158, rabbit polyclonal,1:5000) and Srl214AA (against peptide CFDLADFITKDDFAENL), customized rabbit polyclonal (21st Century Biochemicals, 1:5000). Appropriate HRP-conjugated secondary antibodies (Vector Laboratories) were used at 1:5000). Note that the Srl214AA antibody was verified previously by over-expression, co-migration with epitopetagged Spargel, and use of a second antibody raised against a different Spargel peptide (George \& Jacobs, 2019).

\subsection{Brightfield imaging}

Embryos laid over $24 \mathrm{~h}$ at $25^{\circ} \mathrm{C}$ were collected from matingchamber plates (as above), placed in PBS, rinsed twice in PBS and mounted in PBS onto a slide, for viewing with an Olympus DP73 microscope.

\subsection{Time-lapse imaging of embryonic development}

Embryos laid over $30 \mathrm{~min}$ at $25^{\circ} \mathrm{C}$ were collected from matingchamber plates (as above) and placed in a droplet of halocarbon oil 700 (Sigma-Aldrich) in a 6-well plate. Humidity was maintained by placing a water-soaked cotton-wool plug in adjacent wells. Images were taken every $2 \mathrm{~min}$ for $24 \mathrm{~h}$ or until hatching, using the Cell-IQ live-cell imaging platform (Imagen) at $10 \times$ magnification and a constant $25^{\circ} \mathrm{C}$.

\subsection{Immunohistochemistry and fluorescent imaging}

Batches of approximately 20 ovaries were dissected in PBS, fixed in $4 \%$ paraformaldehyde ( $\mathrm{pH} 7.2)$ on ice for $20 \mathrm{~min}$, rinsed twice with PBT (PBS-0.05\% Tween), washed twice for $15 \mathrm{~min}$ in PBT-BSA (0.5\% BSA) and incubated with primary antibody in PBT-BSA overnight at $4{ }^{\circ} \mathrm{C}$. After two rinses with PBT, samples were washed twice for $15 \mathrm{~min}$ in PBT-BSA then incubated with secondary antibody in PBT-BSA for $2 \mathrm{~h}$ at room temperature. After two further rinses with $\mathrm{PBT}$ and two further washes for $15 \mathrm{~min}$ in PBT-BSA samples were mounted using ProLong ${ }^{\mathrm{TM}}$ Gold Antifade Mountant with DAPI (ThermoFisher Scientific). Embryos laid over $4 \mathrm{~h}$ at $25^{\circ} \mathrm{C}$ were collected from mating-chamber plates (as above), dechorionated using 50\% household bleach (Domestos) and rinsed three times in PBS. $500 \mu \mathrm{l}$ of heptane and $500 \mu \mathrm{l}$ of $4 \%$ paraformaldehyde ( $\mathrm{pH} 7.2$ ) were added and the embryos left to fix over 10-60 min. The aqueous bottom layer was removed, leaving the
Table 1

Densitometric analysis ${ }^{\mathrm{a}}$ of Western blots.

\begin{tabular}{|c|c|c|c|c|c|}
\hline Stage & Protein & Oregon $\mathrm{R}$ & $33914^{\mathrm{b}}$ & $33915^{\mathrm{b}}$ & $\begin{array}{l}\text { Statistical } \\
\text { analysis }^{c}\end{array}$ \\
\hline \multirow[t]{6}{*}{ Ovary $^{\mathrm{d}}$} & Spargel & $1.00 \pm 0.24$ & $1.29 \pm 0.33$ & $0.82 \pm 0.24$ & ns \\
\hline & ATP5A & $1.00 \pm 0.01$ & $0.87 \pm 0.06$ & $0.96 \pm 0.05$ & ns \\
\hline & GAPDH & $1.00 \pm 0.21$ & $0.40 \pm 0.02$ & $0.81 \pm 0.10$ & Oregon R/ \\
\hline & & & & & $33914 *$ \\
\hline & & & & & 33914/ \\
\hline & & & & & $33915^{* *}$ \\
\hline \multirow[t]{4}{*}{ Embryo $^{e}$} & COXIV & $1.00 \pm 0.01$ & $1.46 \pm 0.18$ & nd & ns \\
\hline & ATP5A & $1.00 \pm 0.03$ & $0.78 \pm 0.15$ & nd & ns \\
\hline & NDUFS3 & $1.00 \pm 0.29$ & $0.81 \pm 0.06$ & nd & ns \\
\hline & GAPDH & $1.00 \pm 0.05$ & $1.22 \pm 0.17$ & nd & ns \\
\hline
\end{tabular}

${ }^{a}$ Using Inage $\mathrm{J}$, with normalization to values for Oregon $\mathrm{R}$ for each protein and stage.

b From females/mothers of the indicated RNAi lines crossed to MTD-GAL4.

c Using Student's $t$-test, with Bonferroni correction where appropriate, * $p<0.05, * *-p<0.01$, ns - not significant.

d Based on Western blots shown in Fig. 5C.

e $160 \mathrm{~min}$ AEL, based on Western blots shown in Fig. 6B.

embryos in $500 \mu$ of heptane, to which was added $500 \mu$ of methanol. Embryos were vortexed for $2 \mathrm{~min}$ to remove the vitelline membrane. Heptane, methanol and all material at the interphase was removed, leaving only the devitellinised embryos, which were rinsed three times with methanol, washed three times for $15 \mathrm{~min}$ in PBT-BSA (0.5\% BSA), and processed thereafter as for ovaries. For ovaries the primary antibody used was against ATP5A - Abcam\#14748, 1:5000 and for embryos: Even skipped - DSHB-2B8, Hunchback - Abcam\#197787, Dorsal - DSHB-7A4, all at a dilution of 1:500. Appropriate Alexa Fluor ${ }^{\circledR} 488$ secondary antibodies (Abcam) were used at 1:1000. Images were acquired using a Zeiss LSM800 confocal microscope or, for large-scale embryo size and staining-pattern analysis, with a Hamamatsu S60 nanozoomer WSI digital slide scanner C13210-01 microscope at $5 \times$ magnification. ImageJ software (Regions of Interest tool) was used to calculate the areas of DAPI-stained embryos for estimating their overall size estimation. Embryos stained for localized markers were also examined manually and judged to be positive or negative for the relevant characteristic staining pattern (see Fig. 4).

\subsection{Image processing}

Images have been cropped, rotated and framed for clarity, with addition of scale bars where appropriate, as well as optimized for contrast and brightness, but without other manipulations.

\section{Results}

\section{1. srl expression is developmentally regulated}

Database information on the expression level of $s r l$, based on in situ hybridization and RNA-seq (Berkeley Drosophila Genome Project, Flyatlas) indicates sharp changes in early development and a generally modest expression in adult, which is highest in the ovaries. To compare this expression quantitatively between tissues and stages, we conducted qRT-PCR on RNA extracted from staged early embryos, from each of the larval instars, and from separate sexes from L3 up to adult stage (Fig. 1A, B), using RpL32 RNA as an internal standard. Expression was highest in early embryos (stages 1-3), dropped sharply (at least 10-fold) at stages 4-6 (Fig. 1A), around the time of the mid-blastula transition, rose slightly at L1, then remained low throughout development in both sexes (Fig. 1B). In adults it remained low in males, but was approximately 8-fold higher in females (Fig. 1A), as noted previously (George \& Jacobs, 2019). Dissection of adult females revealed that this sex difference was accounted for by much higher expression in ovaries than in 


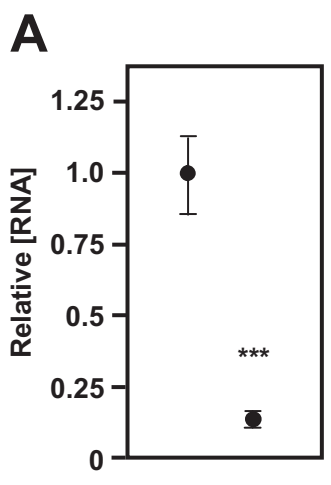

E1-3 E4-6

B

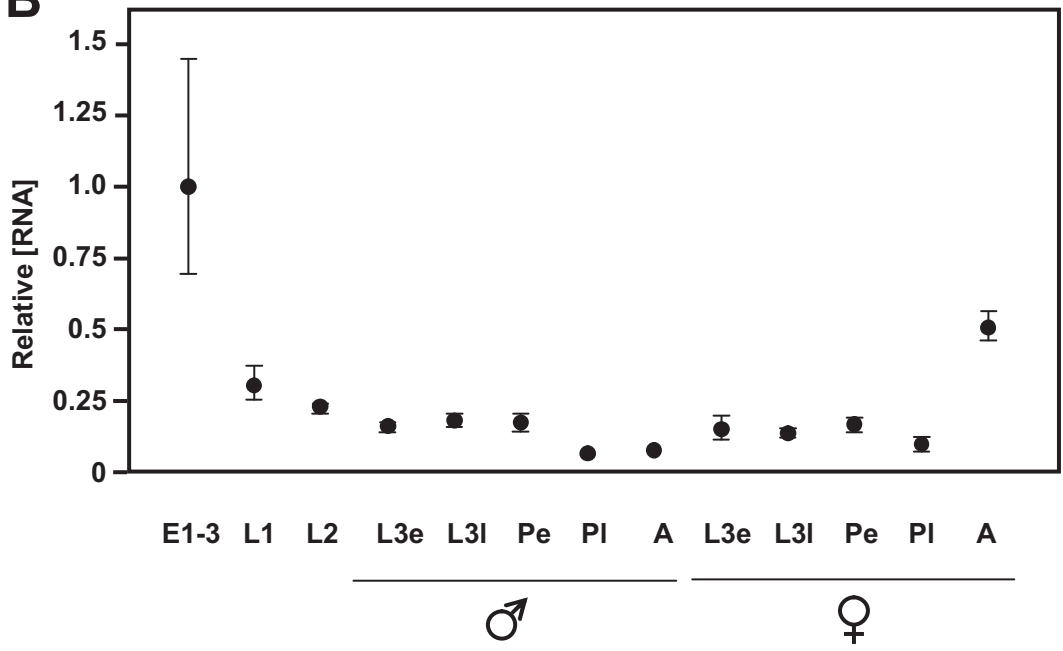

C

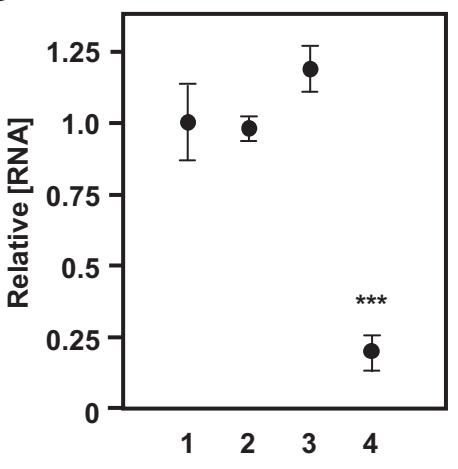

D

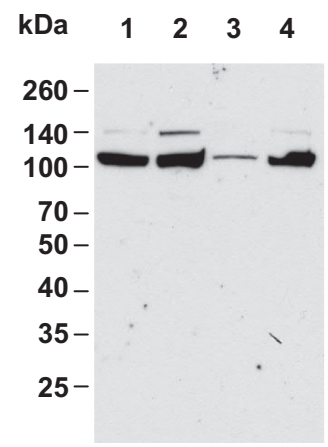

E

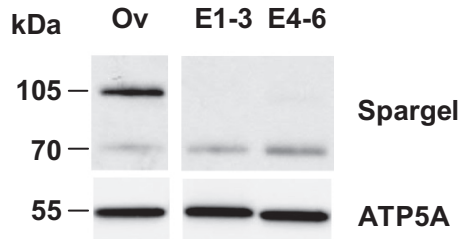

Fig. 1. $s r l$ expression is developmentally regulated.

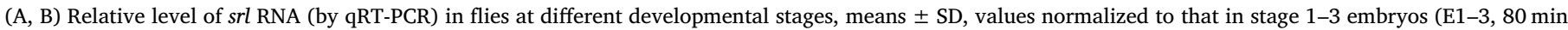

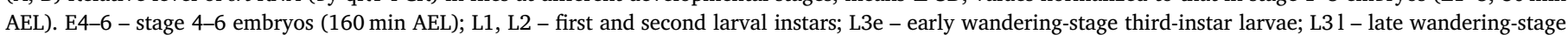

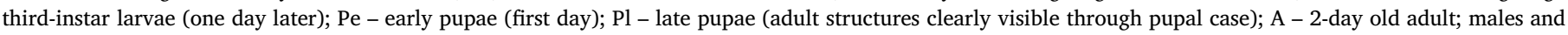

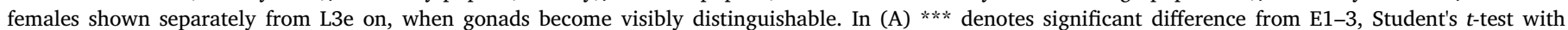

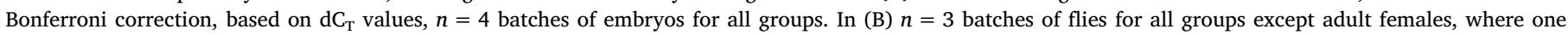

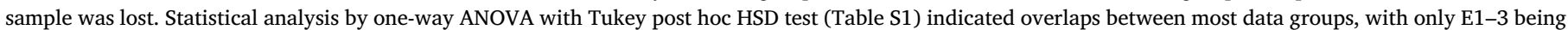

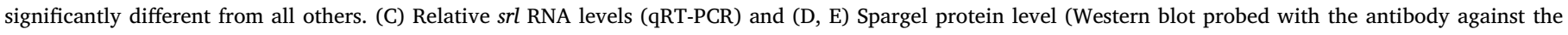

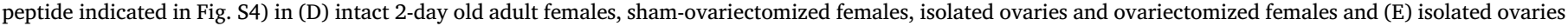

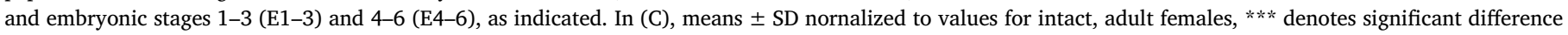

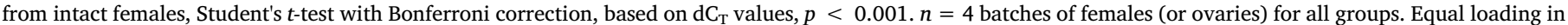

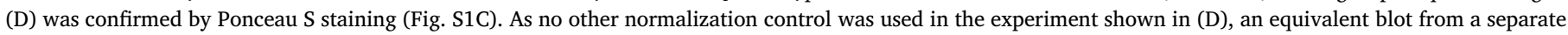

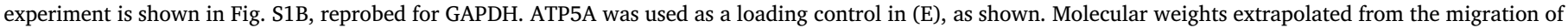

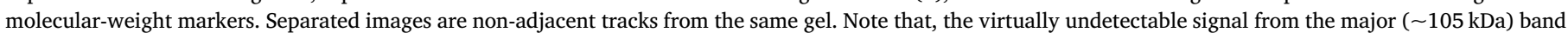

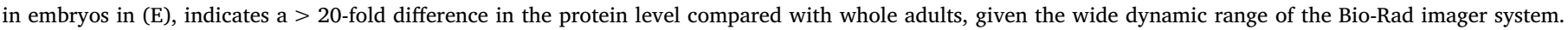

the rest of the adult female (Fig. 1C). We then examined the levels of Spargel protein using a previously verified (George \& Jacobs, 2019), customized antibody (Fig. 1D, S1A, S1B). As noted previously (George \& Jacobs, 2019), the protein was equally represented in males and females (Fig. S1B), despite the disparity in RNA levels. Moreover, in females, the protein was found only at low levels in ovaries, where the RNA is paradoxically most abundant (Fig. 1D, Fig. S1B). Note that samples from ovaries include both germline and somatic (follicle) cells. The $\sim 105 \mathrm{kDa}$ 'main band' was almost undetectable in laid embryos (Fig. 1E), suggesting that, in the ovary, it is mainly of somatic origin.

\subsection{Maternal srl knockdown in the female germline results in embryonic lethality}

To test the effects of downregulating srl expression specifically in the female germline, we made use of the MTD-GAL4 driver, which directs transgene expression in germ cells throughout oogenesis. We combined it with shRNA lines from the Harvard Medical School TriP collection, targeted on srl. We initally tested three such lines. Two of them gave only a weak knockdown of $s r l$ in whole females (Fig. S1D). The third (Bloomington stock center ID 33914, HMS00857, hereafter referred to more simply as line 33914) produced substantial (> 80\%) knockdown (Fig. S1D), and also showed this result when RNA from isolated ovaries was tested (Fig. 2A). One of the lines that showed only a weak knockdown in whole females (Bloomington stock center ID 33915, HMS00858, hereafter referred to as line 33915) did not give knockdown in isolated ovaries either (Fig. 2A), and was used in all subsequent experiments as an additional strain-background negative control, along with wild-type Oregon R. The other negative line (Bloomington stock center ID 57043, GL01019) was not studied further. 

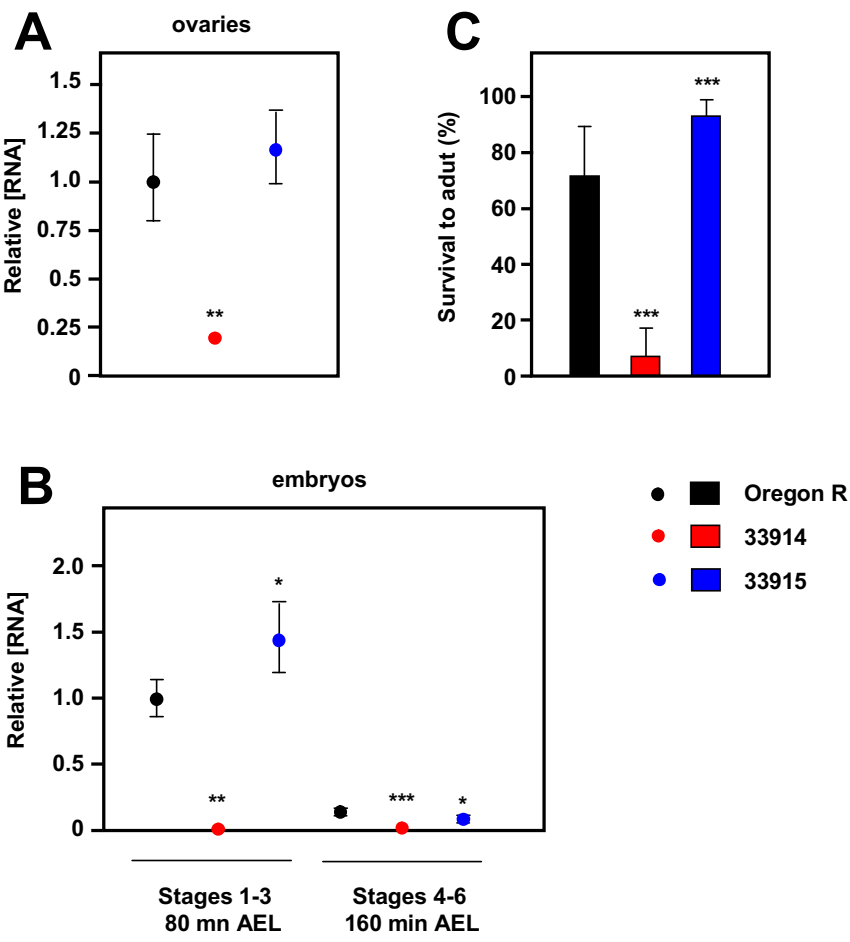

Fig. 2. Germline srl knockdown using the MTD-GAL4 driver.

(A, B) Relative level of $s r l$ RNA (by qRT-PCR) in (A) ovaries of 2-day old females and (B) embryos (stages $1-3$ or 4-6, i.e. 80 or $160 \mathrm{~min}$ AEL, as indicated) from Oregon R (wild-type) females and females of shRNA lines 33914 and 33915, combined with MTD-GAL4 driver; means \pm SD normalized to values for Oregon $\mathrm{R}$ females, $\mathrm{n}=4$ batches of ovaries or embryos for all groups. (C) Survival (egg to adult) for progeny of Oregon R females and females of shRNA lines 33914 and 33915, combined with MTD-GAL4 driver. *, **, *** - significant differences from Oregon R, Student's $t$-test with Bonferroni correction (based on $\mathrm{dC}_{\mathrm{T}}$ values for qRT-PCR), $p<0.05,0.01,0.001$, respectively, $\mathrm{n}=4$ batches of embryos for all groups.

Even when using line 33,914, two nos-GAL4 drivers, NGT40 and NGTA (Tracey Jr et al., 2000; Wheeler et al., 2002) gave no knockdown (Fig. S1D) and were not used further.

The rate of egg-laying by females with germline $s r l$ knockdown (line 33914 combined with the MTD-GAL4 driver) was comparable with wild-type or with line 33915 (Fig. S2A). The embryos that developed manifested a drastically lower ( $>2$ orders of magnitude) level of $\mathrm{srl}$ RNA than controls (Fig. 2B) $80 \mathrm{~min}$ after egg-laying (AEL), and the level remained low at $160 \mathrm{~min}$ AEL, the time when control embryos at stage 4-6 showed a substantial drop in srl RNA abundance (Fig. 2B). Over $90 \%$ of the knockdown embryos failed to complete development (Fig. 2C). However, of the small minority that hatched, almost all developed normally through the larval and pupal stages thereafter, indicating that the developmental defect conferred by maternal $\mathrm{srl}$ knockdown in the germline occurred specifically during embryogenesis. Examination of knockdown embryos laid over a $24 \mathrm{~h}$ period (Fig. 3A) revealed that they varied in their progress through embryogenesis, but most of them failed to reach the later stages in which trachea were visible. Some did not appear to develop at all, and many did not resemble any normal stages, manifesting diverse and typically severe morphological defects. Using time-lapse photography we tracked knockdown embryos through a full 24-hour period. A common pattern (Movie S1) was of grossly abnormal cellularization and gastrulation movements, whilst control embryos cultured in parallel developed as normal (Movie S2).

\subsection{Maternal srl knockdown embryos have an abnormal size distribution}

The embryos laid over a $4 \mathrm{~h}$ period by germline $s r l$ knockdown mothers were analyzed for size and for the expression of genes involved in patterning of the embryo. Whereas control embryos were normally distributed in size (Fig. 3B), those from knockdown mothers showed a broader size distribution, but with a substantial proportion smaller than the size range of controls and a significantly decreased mean size. Automated Gaussian curve fitting failed (Fig. 3B, middle panel), indicating a left-skewed size distribution. The expression of genes in specific embryonic territories in response to localized determinants was, nevertheless, similar to wild-type controls and to published literature, even in small embryos, for the three test gene products studied (Even skipped Fig. 4A, Hunchback - Fig. 4B and Dorsal - Fig. 4C).

\subsection{Germline srl knockdown does not downregulate transcripts related to mitochondrial function}

Given the widely credited view that the main role of the PGC-1 coactivator family is to boost the transcription of genes related to mitochondrial and metabolic function under specific physiological or developmental conditions, the observed embryonic semilethality and morphological abnormalities could be consistent with such a role for $\mathrm{srl}$ in early development (oogenesis and/or embryogenesis). To investigate this possibility, we studied the steady-state levels (normalized to that of cytosolic ribosomal protein RpL32) of transcripts for a range of genes encoding components of the mitochondrial OXPHOS complexes, as well as the major mitochondrial transcription factor and nucleoid protein TFAM, the mitochondrial large-subunit (16S) rRNA, and one reference gene, the Gapdh1 isoform of the glycolytic enzyme GAPDH. In ovaries from germline knockdown females (MTD-GAL4/33914) the levels of these transcripts showed no significant differences from controls (Fig. 5A). In knockdown ovaries we observed ovarian follicles in all stages of development (Fig. 5B), with a normal amount and distribution of DNA and of the mitochondrial OXPHOS protein Bellwether (Drosophila ATP synthase $\alpha$ subunit, ATP5A). By Western blot we also found normal levels of typical OXPHOS proteins such as those detected by antibodies against ATP5A (Fig. 5C, S2C), NDUFS3 (ND-30, Fig. S2C) and COXIV (Fig. S2C), as well as housekeeping proteins $\beta$-actin and histone H4 (Fig. S2C). Against this trend, and despite the level of Gapdh1 mRNA not being significantly less than wild-type (Fig. 5A), we found consistently decreased levels of GAPDH (Fig. 5B, S2C). Surprisingly, and despite the large decrease in srl RNA (Fig. 2A), there were no systematic effects observed on the levels of Spargel polypeptides in ovaries (Fig. 5B). Relative mtDNA levels measured by qPCR were not significantly different from those of Oregon $\mathrm{R}$ wild-type ovaries (Fig. 5C).

In embryos at the $80 \mathrm{~min}$ AEL time point (stage 1-3 in control embryos), we observed increased levels of many of the OXPHOS-related transcripts (Fig. S3A), although in many cases this trend was shared with embryos from MTD-GAL4/33915 mothers, so may be a background effect. The steady-state level of ATP5A (Bellwether) protein was unchanged from controls (Fig. S3B), whilst that of Spargel polypeptides was extremely low, as in controls (Fig. S3B). mtDNA copy number, although more varied than in controls at this time-point (Fig. S3D), was of a similar order of magnitude. More pronounced changes were seen at the $160 \mathrm{~min}$ AEL time-point (stage 4-6 in controls), following the period when $s r l$ mRNA is normally at maximal abundance, but then declines (Fig. 1A), and when development in embryos from knockdown mothers becomes clearly aberrant (Supplementary Movie S1). However, here again the trend from $s r l$ knockdown was towards increased, not decreased levels of OXPHOS-related transcripts (Fig. 6A), which were clearly elevated compared with both Oregon R and MTD-GAL4/ 33915 controls. Typical OXPHOS proteins were unchanged compared with wild-type control embryos (Fig. 6B), and this now applied also to GAPDH (Fig. 6B). Relative mtDNA copy number (Fig. 6C, S3D) declined 

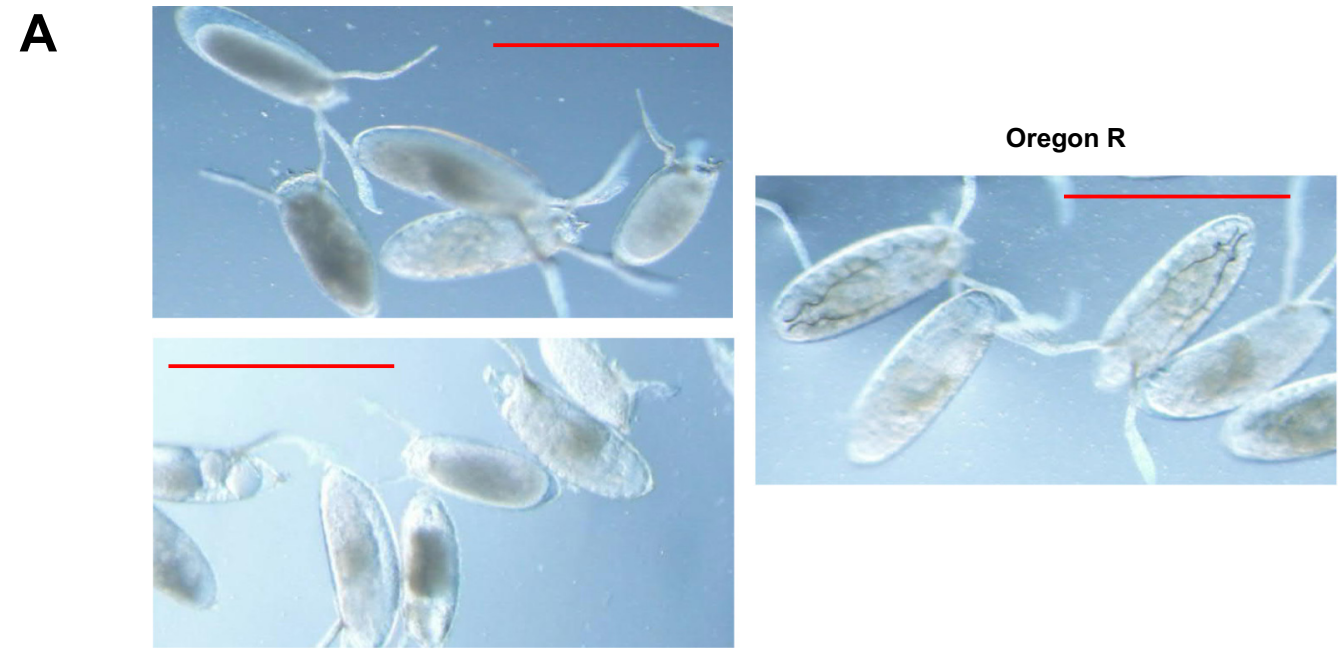

B
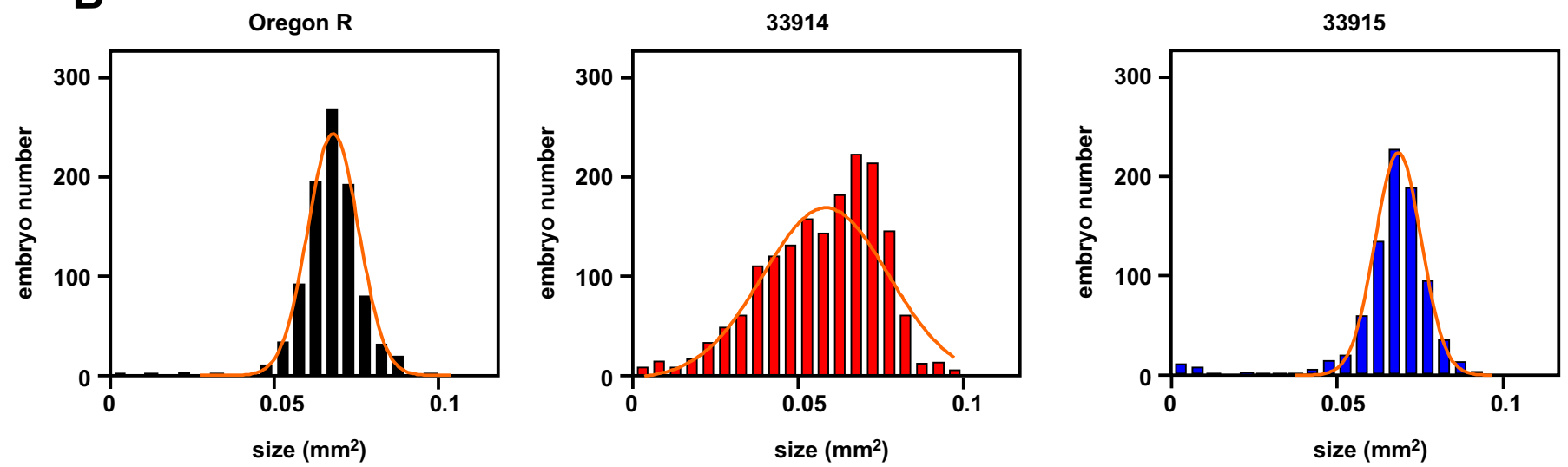

Fig. 3. Germline srl knockdown results in abnormal size distribution of embryos.

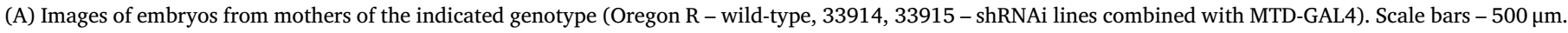

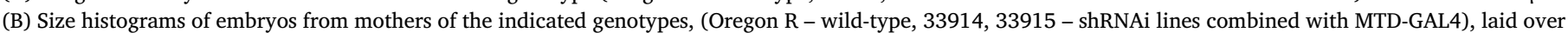

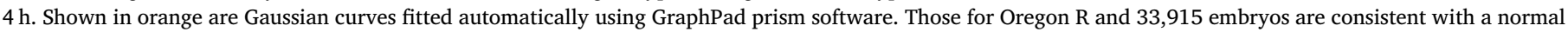

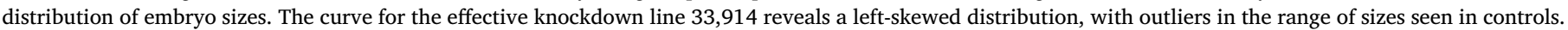

substantially between the two embryo time-points, in line with the expected synthesis of nuclear DNA. Although copy number in maternal srl knockdown (MTD-GAL4/33914) embryos remained slightly higher than in wild-type (Oregon R), this was shared with the MTD-GAL4/ 33915 control (Fig. 6C, S3D). This indicates that it was most likely a background effect, possibly related to the timing of the final rounds of nuclear DNA synthesis.

\section{Discussion}

In this study we demonstrated that $s r l$ function in the germline is required to ensure successful embryogenesis. In summary, gerrmline $\mathrm{srl}$ knockdown produced embryos with an abnormal size distribution (Fig. 3B) and decreased mean size, which failed to develop normally (Fig. 3A) and exhibited aberrant patterns of cellularization and gastrulation movements that did not resemble any normal developmental stage (Movie S1). However, knockdown produced no detectable abnormalities in the embryonic expression of axial specifiers dependent on localized determinants laid down during oogenesis (Fig. 4) and no significant changes in mtDNA copy number (Figs. 5D, 6C) or OXPHOS proteins (Figs. 5C, 6B). mRNAs for OXPHOS subunits were unchanged in oocytes (Fig. 5A) but generally elevated 160 min AEL (Fig. 6A) Although $s r l$ was at its highest levels in early embryos and in ovaries,
Spargel protein was paradoxically at very low levels in these developmental stages (Fig. 1), and was not affected by germline knockdown of the RNA. We here discuss the significance and implications of these findings.

\subsection{Respective roles of spargel RNA and protein in early development}

Two different antibodies raised against non-overlapping peptides from the Spargel protein detect the same bands in Western blots (George \& Jacobs, 2019), namely a major isoform migrating at an apparent molecular weight of $\sim 105 \mathrm{kDa}$ and a less abundant isoform migrating at $\sim 120 \mathrm{kDa}$. The same bands were detected in S2 cells transfected with an epitope-tagged variant of $s r l$, probed for the epitope tag (George \& Jacobs, 2019). In the present study we also detected a minor band in ovaries (Fig. 5C) and embryos (Fig. S3B), visible only at long exposure, migrating around $\sim 70 \mathrm{kDa}$. Based on the previous data (George \& Jacobs, 2019) we consider that these antibodies are reliable for detecting Spargel polypeptides, although not necessarily all Spargel polypeptides. Any Spargel isoforms in which the two relevant (nonoverlapping) peptides used for raising antibodies (see Fig. S4) are both excised or heavily modified would remain undetected. The primary translation products of both of the reported $s r l$ mRNA splice variants (see FlyBase (Thurmond et al., 2019; Chintapalli et al., 2007)) differ by 
A

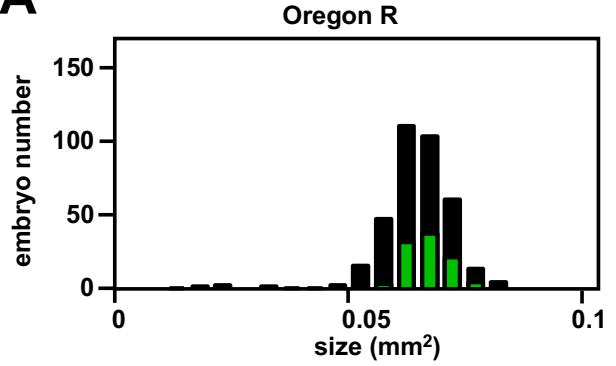

B

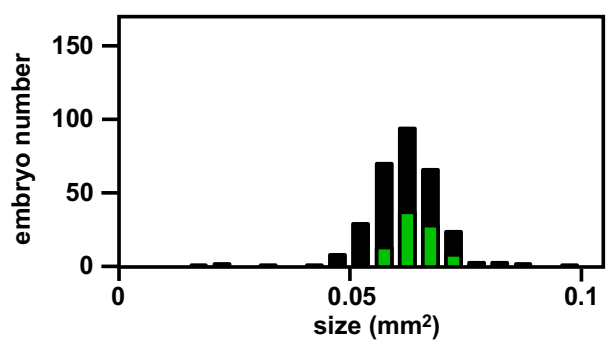

C

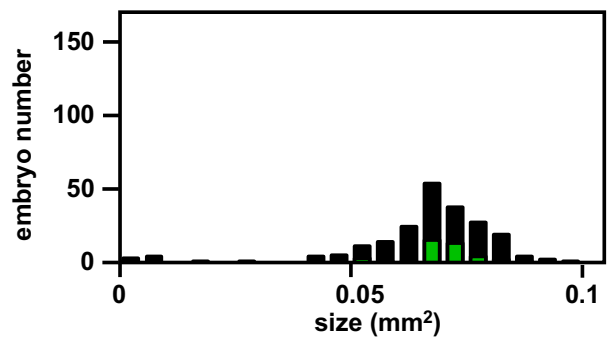

33914

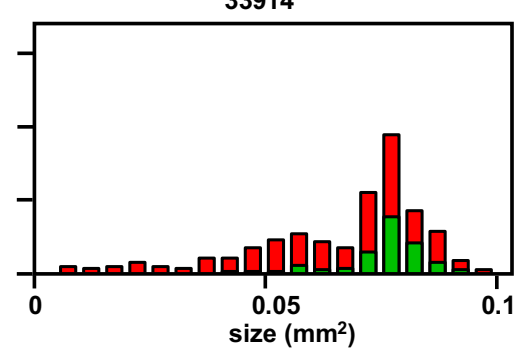

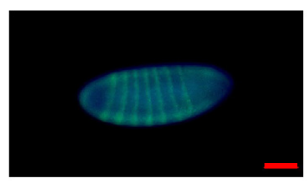

Oregon $\mathbf{R}$ even skipped

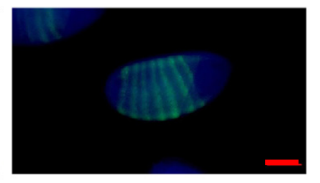

33914

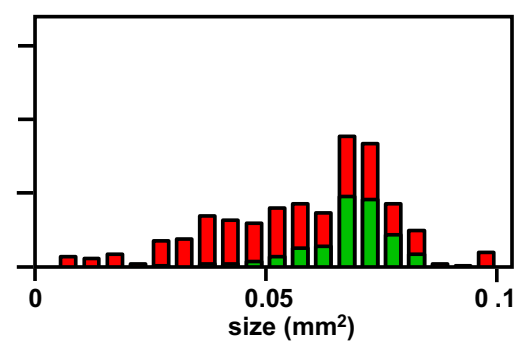

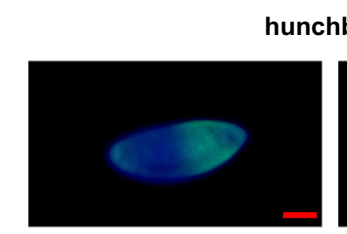

Oregon $\mathbf{R}$ unchback

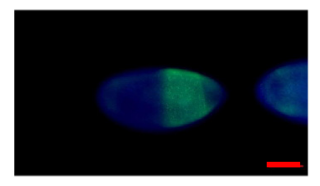

33914

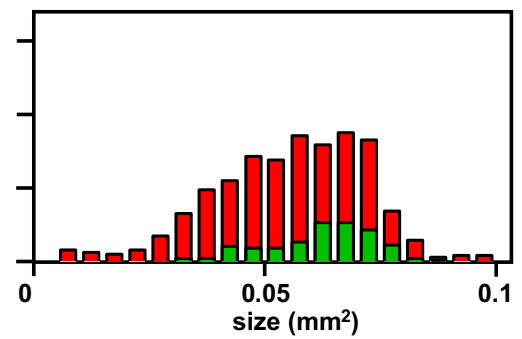

Fig. 4. Germline $s r l$ expression does not alter embryonic expression of localized determinants.

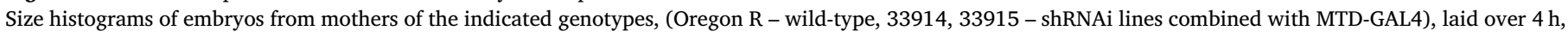

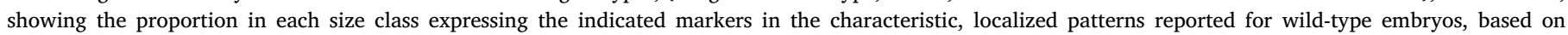

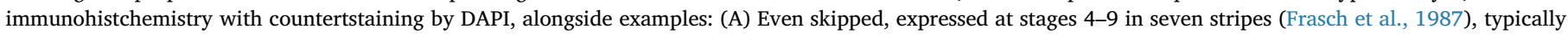

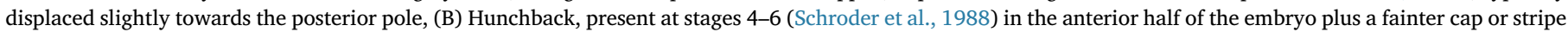

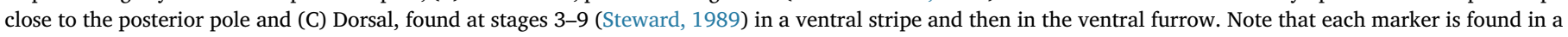
normal pattern in roughly the expected proportion of 33914 ( $s r l$ knockdown) embryos of different size classes. Scale bars - $100 \mu$ m.

only a short run of amino acids near the N-terminal region (Fig. S4), are each predicted to be detected by these antibodies. Several observations suggest that we may be missing some vital information, which could relate to one or more isoforms of the protein that are not detected by either of these antibodies, or to a role for srl RNA that is distinct from its coding function as the mRNA for Spargel. First and most puzzling is the discordant result regarding the abundance of $s r l$ RNA and Spargel protein. The RNA was several-fold higher in females than in males, but the difference is accounted for by its much higher representation in the ovaries than in somatic tissues. Ovariectomized females had similar amounts of the RNA as males (Fig. 1B, C). However, the major, $\sim 105 \mathrm{kDa}$ protein isoform follows a distinct, in some ways opposite pattern, being present in similar amounts in adult males and ovariectomized females, but at much lower levels in ovaries. In the early embryo, the RNA was at its highest level of any stage (Fig. 1A), yet the major Spargel protein band of $\sim 105 \mathrm{kDa}$ was virtually undetectable, with only the minor $\sim 70 \mathrm{kDa}$ band clearly visible. Further, despite effective RNAi knockdown of $s r l$ at the RNA level (Fig. 2), the protein level was essentially unaffected, at least in ovaries (Fig. 5C).

It should be noted that different normalization standards were used for RNA (RpL32) and for protein (GAPDH). For RNA normalization $R p L 32$ is commonly used as reference gene, making our data comparable with that of other studies. For protein normalization, there is no fully agreed universal standard, although GAPDH is widely used, and shows only minor fluctuations in most contexts. RpL32 has not been widely used as a protein standard in Drosophila. We considered the effects of applying the same standard for normalization to both RNA and protein. The abundance of Gapdh1 mRNA (relative to that for RpL32) showed no significant differences between the genotypes in ovary (Fig. 5A), nor in embryos 80 min AEL (Fig. S3A). Moreover, based on the data in Flyatlas (Thurmond et al., 2019; Chintapalli et al., 2007), the abundance of Gapdh1 and RpL32 mRNAs in ovary is at a very similar ratio to that in the whole fly (0.76 and 0.86 , respectively). Therefore, switching to Gapdh1 as an alternative normalization standard would make no difference to the overall result for those stages, nor to the major finding of radically different $s r l$ mRNA and protein abundances. It should also be noted that, at $160 \mathrm{~min}$ AEL, there is a sharp drop in Gapdh1 mRNA abundance in all genotypes (Fig. 6A), making it unsuitable as a reference 'housekeeping' mRNA during the specific context of embryogenesis where, in any case, new protein synthesis contributes little to the steady-state level of abundant housekeeping proteins such as GAPDH.

There is no reported evidence for an antisense srl RNA or any truncated RNA lacking the start codon, from the $s r l$ locus. Therefore, the RNAi target in our experiments must be one or all of the reported $s r$ mRNA isoforms. One possible explanation for our findings would be that the Spargel protein detected in ovaries and embryos does not originate in the germline but is imported from follicle cells, whilst the 


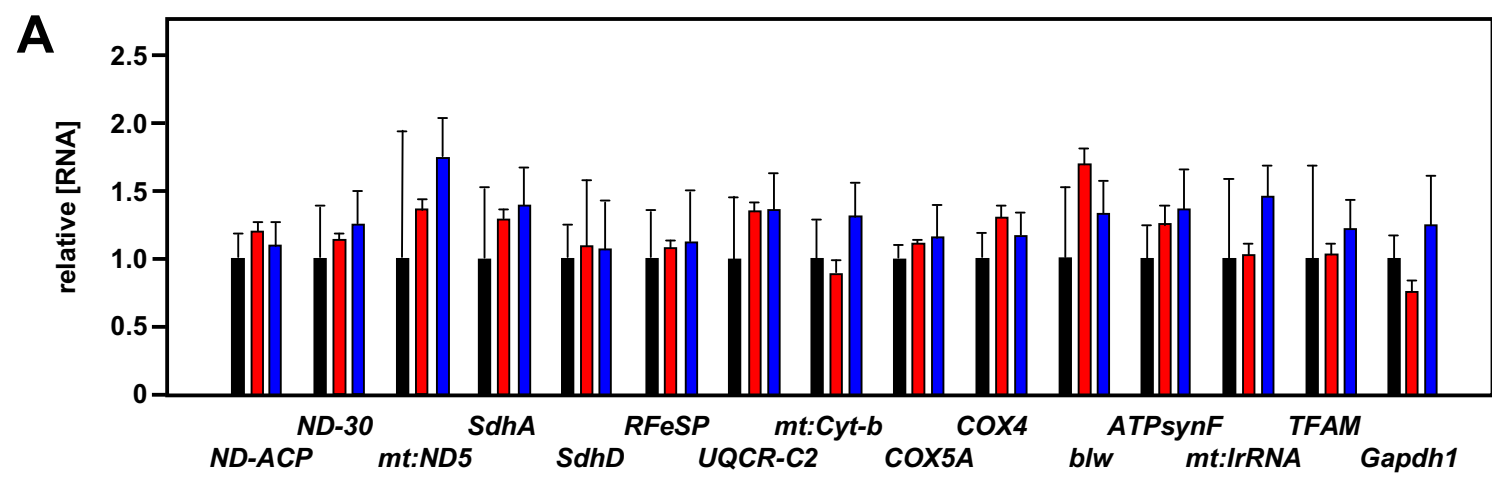

B
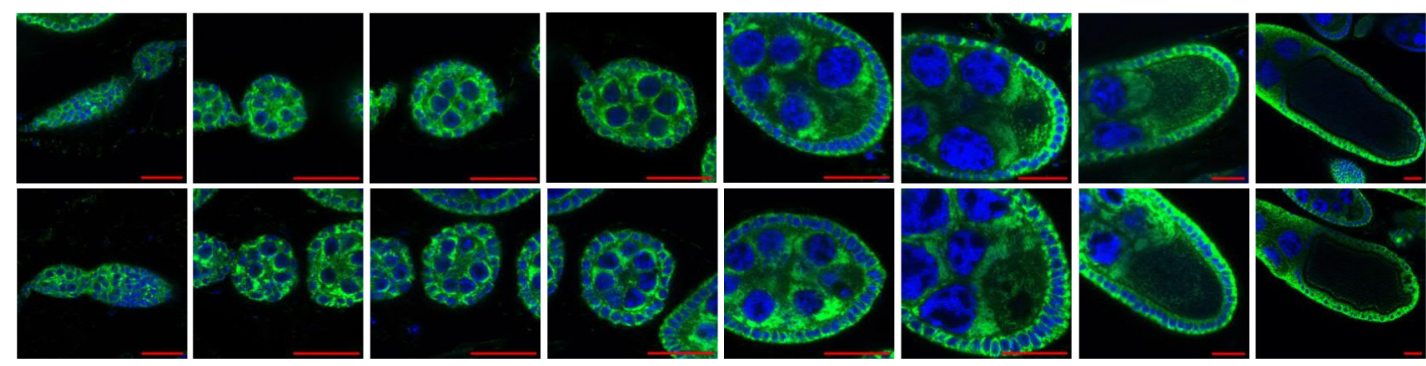

Oregon R

- Oregon $\mathbf{R}$

( 33914

33915
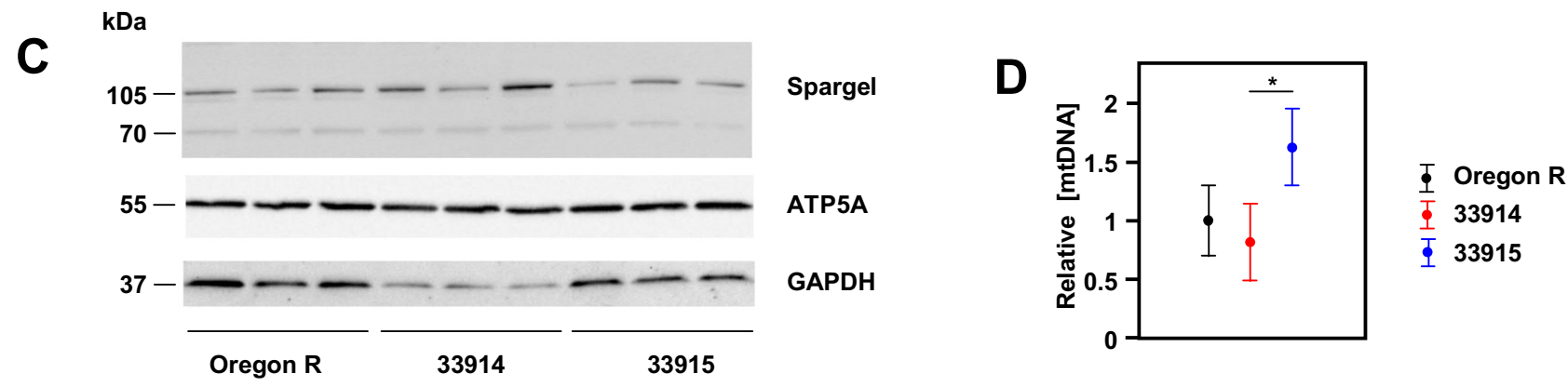

Fig. 5. Effect of germline srl knockdown on RNA, protein and mtDNA levels in ovaries.

(A) qRT-PCR measurements of RNA levels of the indicated genes (symbols as in FlyBase (Thurmond et al., 2019; Chintapalli et al., 2007)) in ovaries from females of the indicated genotypes: Oregon R (wild-type), 33914, 33915 - shRNA lines 33914 and 33915 in combination with MTD-GAL4. Means \pm SD, n $=4$ batches of ovaries for each group. There were no significant differences of the other groups from Oregon $\mathrm{R}$ (Student's $t$-test with Bonferroni correction using $\mathrm{dC}_{\mathrm{T}}$ values). Note that, because fold-differences rather than $\mathrm{dC}_{\mathrm{T}}$ values are plotted, SDs in the two directions are unequal. For clarity, only the larger SD, above the mean, is shown. (B) Images of ovarian follicles at various stages of oogenesis (left to right: germarium, stages 1-6, 10 and 11 or later), from females of the indicated genotypes, stained as shown. Scale bars $=20 \mu \mathrm{m}$. (C) Western blots of protein extracts from ovaries of females of the indicated genotypes, probed as indicated. Molecular weights extrapolated from the migration of molecular-weight markers. The images are from different gels of the same protein samples. See Fig. S2B, for a representative Ponceau S-stained membrane confirming equal protein loading. Densitometric signal analysis, normalized to values for Oregon $R$, is shown in Table 1. (D) Relative mtDNA copy numbers in ovaries of females of the indicated genotypes, normalized to Oregon R, means \pm SD, $n \geq 4$ for each group. * indicates significant differences between maternal genotypes (one-way ANOVA with Tukey post hoc HSD test, based on $\mathrm{dC}_{\mathrm{T}}$ values, $p<0.05$ ). For full statistical analysis on all copynumber measurements, i.e. both in ovaries and embryos, using two-way ANOVA, see Table S2.

abundant srl mRNA in the oocyte and early embryo is untranslated, and has a novel but unknown (non-coding) role in early development.

Like PGC- $1 \alpha$ in mammals, $s r l$ is known to be regulated post-translationally by RNF34-mediated ubiquitylation (Wei et al., 2018). In adult muscle, knockdown of srl counteracts the metabolic and physiological effects of RNF34 knockdown (Wei et al., 2018), including the promotion of mitochondrial biogenesis. Translational regulation, as hypothesized previously (George \& Jacobs, 2019) might account for low levels of the protein despite high levels of the RNA, if $s r l$ translation is responsive to levels of the encoded protein, as documented for a subset of genes in yeast (Beyer et al., 2004). Although purely speculative at this point, such an idea needs to be thoroughly investigated.

\subsection{Maternal spargel is required in the germline for embryo viability}

In this study, we showed that the female sterility (Tiefenböck et al.,
2010) or near sterility (Mukherjee et al., 2014) previously seen in $s l^{1}$ flies is due to an effect specifically in the female germline, affecting the viability of the resulting embryos. Previous studies indicated a delay in oogenesis in the $\operatorname{srl}^{1}$ hypomorph (Mukherjee et al., 2014), although we did not find any difference in fecundity as a result of germline srl knockdown (Fig. S2A), nor any obvious difference in ovarian morphology (Fig. 4). The previous findings may therefore have been influenced by somatic insufficiency of $s r l$ (e.g. in the fat body). Here we showed that $s r l$ knockdown in the germline affects embryo size (Fig. 3) and produces a dramatic, disruptive effect on embryonic development, commencing around the time of the mid-blastula transition (Movie S1). Nevertheless, we found no specific evidence of an effect on mitochondria.

Gametogenesis clearly does depends upon mitochondrial function in somatic cells of the developing gonad (Reeve et al., 2007), although spatial and temporal disruption of mitochondrial movement into the 


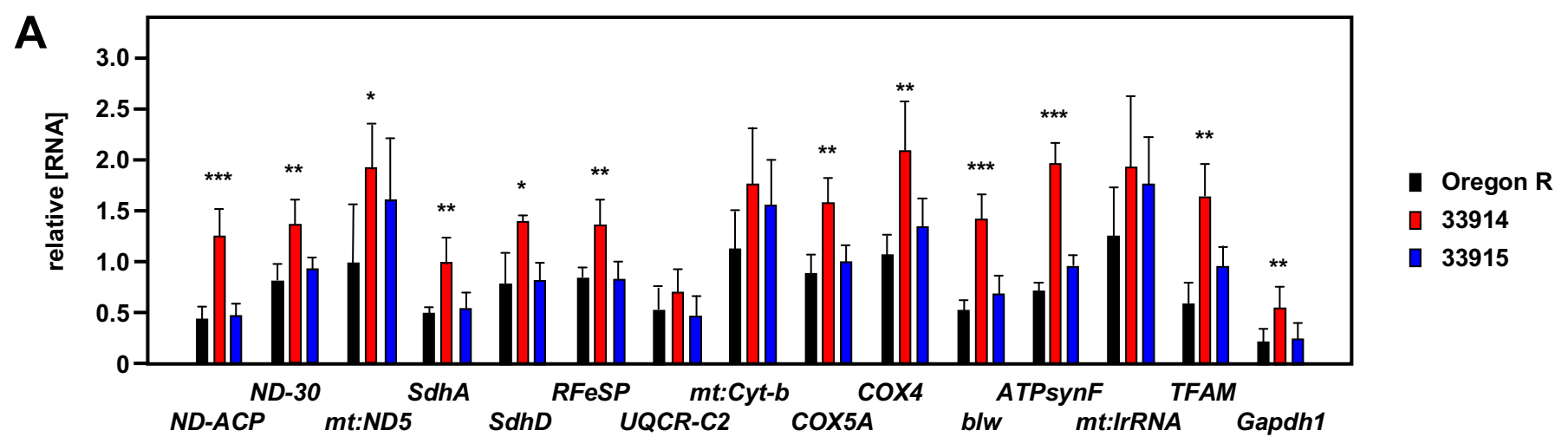

B

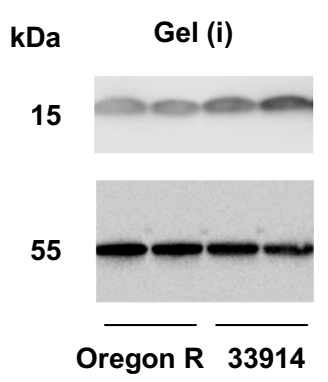

Gel (ii)

COXIV

ATP5A

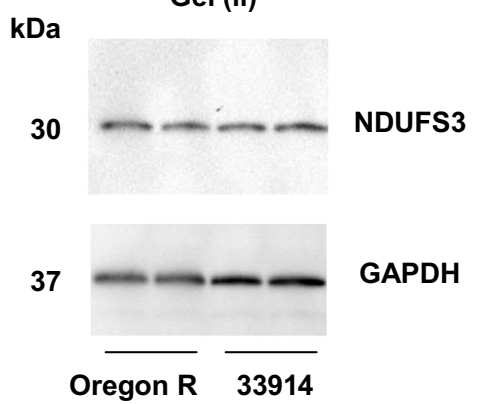

C

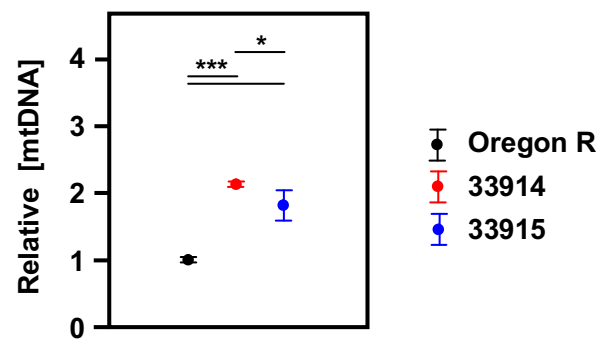

Fig. 6. Effect of germline $s r l$ knockdown on RNA, protein and mtDNA levels in embryos.

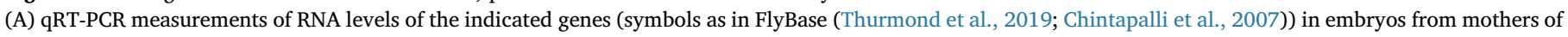

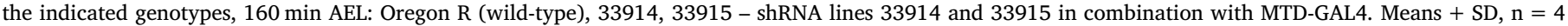

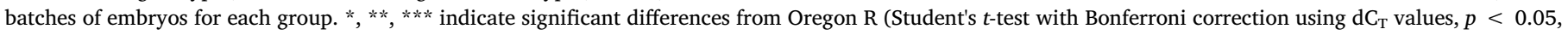

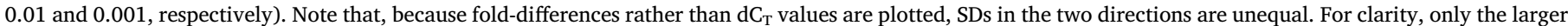

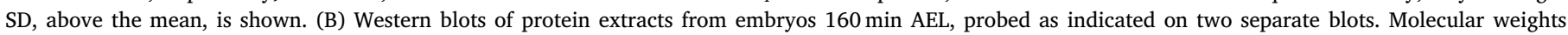

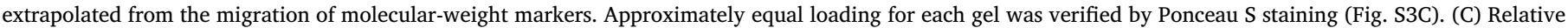

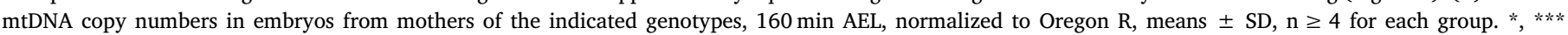

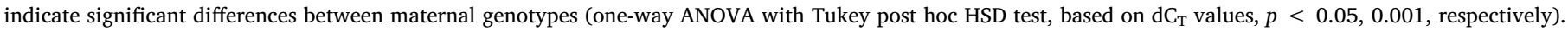
For full statistical analysis on all copy-number measurements, i.e. both in ovaries and embryos, using two-way ANOVA, see Table S2.

oocyte from the nurse cells does not prevent the development of viable progeny (Cox \& Spradling, 2006).

Mitochondria are a major component of the Balbiani body (Cox \& Spradling, 2003), which is involved in the formation of polar granules, the germ plasm (Lehmann, 2016) that specifies the future germline. The widespread and dramatic effects on cellularization are not consistent with any specific effect on future germ cells, although we cannot exclude the possibility that components of the germ plasm may have been incorrectly distributed to other regions of the srl knockdown oocyte, where they might interfere with nuclear re-localization or specific aspects of fate specification during blastoderm formation and/or cellularization.

Several lines of argument support the conclusion that the embryonic lethality was due to a genuine RNAi effect, despite the lack of correspondence between $s r l$ RNA and protein levels, as detected by the antibody. First, knockdown was highly effective at the RNA level, whereas other RNAi lines targeted against srl gave no such knockdown and also no observable phenotype. Second, the previously studied hypomorph $\operatorname{srl}^{1}$ also showed female sterility (Tiefenböck et al., 2010), even though it was not possible to establish whether this was a somatic or germlinespecific effect. The similar phenotype makes it highly unlikely that our findings are an off-target effect. Although an off-target effect cannot be completely ruled out, the usual way of excluding it, which would be to recode the protein and express it in the RNAi background, would be uninterpretable, given the non-congruence of the RNA and protein data. Note also that massive overexpression at the RNA level also had only modest effects on the protein (George \& Jacobs, 2019). Thus, insufficiency of the srl RNA as such, rather than of the encoded protein, may underlie the embryonic phenotype, which would impute an undefined, non-coding function to the RNA.

\subsection{Does size matter?}

$s r l$ knockdown embryos were smaller than their wild-type or control counterparts (Figs. 3, 4), and their sizes spanned a greater range. However, many were in the normal size range, despite the fact that only $\sim 10 \%$ of them were able to complete development, implying that size is not the sole determinant of developmental success or failure following srl knockdown. Rather, the abnormal size distribution should be considered an indicator of unknown aberrations during oogenesis. Oogenesis was not grossly disturbed (Fig. 5B), and knockdown embryos were ostensibly normal as regards localized determinants (Fig. 4). Compared with controls, the amount of mtDNA relative to nuclear DNA in knockdown ovaries or embryos showed only minor variations that are probably attributable to strain background (Fig. 6D, S3D). Once cellularization was complete (160 min AEL), relative mtDNA copy number was only slightly elevated compared with controls (Fig. 6C, S3D), implying that total nuclear DNA synthesis during embryogenesis 
was close to normal. The only consistent differences we could detect in knockdown ovaries was in the representation of GAPDH, which was decreased (Fig. 5C, S2C).

Our tentative interpretation is that a sufficient level of spargel gene product is required to ensure the supply of one or more vital components to the oocyte. Noting that the qRT-PCR data at both the $80 \mathrm{~min}$ AEL (Fig. S3A) and 160 min AEL (Fig. 6A) time-points has been normalized to the values for each transcript in Oregon R embryos at $80 \mathrm{~min}$ AEL, it is evident that there is a general decline in most maternal transcripts (compared with RpL32) between these two time-points in control embryos. Yet in knockdown embryos this decline did not occur (Fig. 6A), suggesting a global failure of the transcriptional activation that occurs once the cleavage cycles of nuclear division are complete (Anderson \& Lengyel, 1979). This fits the canonical activity of Spargel as a transcriptional coactivator, and would imply that one of its roles in the embryo could be to boost new transcription across the genome, at cellularization. Note, however, that the zygotic activation of evenskipped and hunchback transcription during the syncitial phase of embryonic development occurred normally (Fig. 4A, B). These early switches in transcription involve specific mechanisms, such as the dilution of maternally synthesized repressors (Pritchard \& Schubiger, 1996), and may be independent of a general requirement for Spargel. The fact that GAPDH protein was decreased in knockdown ovaries (Fig. 5C) also suggests the possibility that Spargel may indeed be acting as a transcriptional coactivator during oogenesis, but with a completely different set of specific targets than those related to mitochondria. A completely different possibility would be that spargel has a more specific role in cellularization, which might directly involve the srl RNA, which abruptly declines in abundance in control embryos around this time (Fig. 1), rather than the protein.

\subsection{Ancestral function(s) of PGC-1 family coactivators}

In mammals, only one member of the PGC-1 family, PPRC1, is an essential gene, being specifically required for early development in the mouse (He et al., 2012), as well as for the growth program in somatic cells (Andersson \& Scarpulla, 2001; Vercauteren et al., 2009). Its functions are less well characterized than those of PGC-1 $\alpha$ and PGC-1 $\beta$. Although PPRC1 is responsive to mitochondrial metabolic stress, which it counteracts (Gleyzer \& Scarpulla, 2011), it is less obviously focused on metabolism than PGC- $1 \alpha$ and PGC-1 $\beta$. For this reason, it has been widely assumed that it arose by duplication and diversification, evolving novel functions in the process. The fact that the PGC-1 family is represented in Drosophila by a single gene offered a theoretical opportunity to identify the ancestral function of the gene family, and re-assess the meaning of the gene family in mammals.

Previous studies of $s r l$ have suggested a relationship with metabolism, although the hypomorph $s{ }^{1}$ was earlier found to have a growth defect and to exhibit female sterility (Tiefenböck et al., 2010), with a few 'escapers' (Mukherjee et al., 2014). Using a highly specific driver/ RNAi vector combination, our studies have now shown that this essential function is exercised in the female germline and/or early embryo, but does not involve any specific effect on mitochondria or on gene expression connected to metabolism.

The developmental expression pattern of PPRC1 shares some similarities with that of $s r l$, being abundant during the cleavage stages, or when embryonic stem cells are induced to form embryoid bodies, but then falling away sharply (He et al., 2012). These various features suggest that $s r l$ has more in common with PPRC1 than with other members of the PGC-1 family, and that these shared properties may be ancestral. On the other hand, previous data relating srl to growth regulation and responsiveness to insulin signalling (Tiefenböck et al., 2010; Mukherjee \& Duttaroy, 2013), imply that the ancestral PGC-1 coactivator may have performed functions both in early development and in metabolic regulation of growth, and these only diverged functionally later on in mammals.

\subsection{Clinical relevance}

In humans, the PGC-1 coactivators have been implicated in key physiological and pathological processes linked to diet and metabolism (Cheng et al., 2018). Moreover, their upregulation has been shown to be beneficial in numerous disease models (Cheng et al., 2018), and they have been proposed as potential drug targets via the use of agents such as bezafibrate, that act as PGC-1 pan-agonists (Komen \& Thorburn, 2014). However, such trials have so far met with only limited success (Orngreen et al., 2015). The present study indicates one fundamental reason why this might be the case: namely, that, in addition to their roles in energetic homeostasis, the PGC-1 coactivators are also involved in regulating other processes, including key events in early development performed in Drosophila by $s r l$ and in mammals by PPRC1. Therefore, PGC-1 pan-agonists may simply have too broad an effect, with unintended side effects and self-cancelling modifications of metabolism, unless some way can be found to target PGC-1 regulation of metabolic functions more specifically.

\subsection{Conclusions}

In conclusion, whilst spargel expression in the female germline plays an important function in reproductive viability, there is no evidence supporting mitochondrial biogenesis or metabolic homeostasis as the object of that function. Instead, spargel potentially plays a more global role in oogenesis, ensuring adequate resources for the developing embryo after oviposition. We suggest that the PGC-1 coactivator family might better be considered as supplying a boost to transcription in response to several different types of metabolic, developmental or physiological signalling, and that the precise targets of this effect depend more on the tissue and context than on specific properties of the coactivators.

Supplementary data to this article can be found online at https:// doi.org/10.1016/j.mito.2019.08.006.

\section{Authors' contributions}

HTJ and JG jointly conceived the study. JG performed the experiments and analyses and produced the first draft of the figures. HTJ supervised the work, finalized the figures, and drafted the manuscript, which was finalized by both authors.

\section{Funding}

This work was supported by the Academy of Finland [grant numbers 283157 and 272376]; Tampere University Hospital Medical Research Fund and the Sigrid Juselius Foundation. The funders played no part in the experimental work or compilation of the manuscript.

\section{Data statement}

All data relevant to the work presented here are available from the paper itself or its supplementary files, and may be freely used for academic purposes.

\section{Declaration of Competing Interest}

The authors declare that they have no competing interests.

\section{Acknowledgements}

We thank Tea Tuomela and Eveliina Teeri for technical assistance, Eric Dufour for advice and many useful discussions and Troy Faithfull for critical reading of the manuscript. 


\section{References}

Adán, C., Matsushima, Y., Hernández-Sierra, R., Marco-Ferreres, R., Fernández-Moreno, M.A., González-Vioque, E., et al., 2008. Mitochondrial transcription factor B2 is essential for metabolic function in Drosophila melanogaster development. J. Biol. Chem. 283, 12333-12342.

Akiyama, T., Okada, M., 1992. Spatial and developmental changes in the respiratory activity of mitochondria in early Drosophila embryos. Development. 115, 1175-1182.

Anderson, K.V., Lengyel, J.A., 1979. 1979. Rates of synthesis of major classes of RNA in Drosophila embryos. Dev. Biol. 70, 217-231.

Andersson, U., Scarpulla, R.C., 2001. Pgc-1-related coactivator, a novel, serum-inducible coactivator of nuclear respiratory factor 1-dependent transcription in mammalian cells. Mol. Cell. Biol. 21, 3738-3749.

Beyer, A., Hollunder, J., Nasheuer, H.P., Wilhelm, T., 2004. Post-transcriptional expression regulation in the yeast Saccharomyces cerevisiae on a genomic scale. Mol. Cell. Proteomics 3, 1083-1092.

Cheng, C.F., Ku, H.C., Lin, H., 2018. PGC-1 $\alpha$ as a pivotal factor in lipid and metabolic regulation. Int. J. Mol. Sci. 19, 3447.

Chintapalli, V.R., Wang, J., Dow, J.A.T., 2007. Using FlyAtlas to identify better Drosophila models of human disease. Nat. Genet. 39, 715-720.

Cox, R.T., Spradling, A.C., 2003. A Balbiani body and the fusome mediate mitochondrial inheritance during Drosophila oogenesis. Development. 130, 1579-1590.

Cox, R.T., Spradling, A.C., 2006. Milton controls the early acquisition of mitochondria by Drosophila oocytes. Development. 133, 3371-3377.

Finck, B.N., Kelly, D.P., 2006. PGC-1 coactivators: inducible regulators of energy metabolism in health and disease. J. Clin. Invest. 116, 615-622.

Frasch, M., Hoey, T., Rushlow, C., Doyle, H., Levine, M., 1987. Characterization and localization of the even-skipped protein of Drosophila. EMBO J. 6, 749-759.

Galloni, M., 2003. Bonsaï, a ribosomal protein S15 homolog, involved in gut mitochondrial activity and systemic growth. Dev. Biol. 264, 482-494.

George, J., Jacobs, H.T., 2019. Minimal effects of spargel (PGC-1 $\alpha$ ) overexpression in a Drosophila mitochondrial disease model. Biol. Open. https://doi.org/10.1242/bio. 042135. (in press).

Gleyzer, N., Scarpulla, R.C., 2011. PGC-1-related coactivator (PRC), a sensor of metabolic stress, orchestrates a redox-sensitive program of inflammatory gene expression. J. Biol. Chem. 286, 39715-39725.

He, X., Sun, C., Wang, F., Shan, A., Guo, T., Gu, W., Cui, B., Ning, G., 2012. Peri-implantation lethality in mice lacking the PGC-1-related coactivator protein. Dev. Dyn. 241, 975-983.

Thurmond, J., Goodman, J.L., Strelets, V.B., Attrill, H., Gramates, L.S., Marygold, S.J., Matthews, B.B., Millburn, G., Antonazzo, G., Trovisco, V., Kaufman, T.C., Calvi, B.R., the FlyBase Consortium, 2019. FlyBase 2.0: the next generation. Nucleic Acids Res 47 (D1), D759-D765.

Hudson, A.M., Cooley, L., 2014. Methods for studying oogenesis. Methods. 68, 207-217.

Kemppainen, E., George, J., Garipler, G., Tuomela, T., Kiviranta, E., Soga, T., Dunn, C.D., Jacobs, H.T., 2016. Mitochondrial dysfunction plus high-sugar diet provokes a metabolic crisis that inhibits growth. PLoS One 11, e0145836.

Komen, J.C., Thorburn, D.R., 2014. Turn up the power - pharmacological activation of mitochondrial biogenesis in mouse models. Br. J. Pharmacol. 171, 1818-1836. Lehmann, R., 2016. Germ plasm biogenesis-an Oskar-centric perspective. Curr. Top. Dev. Biol. 116, 679-707.

Mukherjee, S., Duttaroy, A., 2013. Spargel/dPGC-1 is a new downstream effector in the insulin-TOR signaling pathway in Drosophila. Genetics. 195, 433-441.

Mukherjee, S., Basar, M.A., Davis, C., 2014. Duttaroy. Emerging functional similarities and divergences between Drosophila Spargel/dPGC-1 and mammalian PGC-1 protein. Front. Genet. 5, 216.

Ni, J.Q., Zhou, R., Czech, B., Liu, L.P., Holderbaum, L., Yang-Zhou, D., et al., 2011. A genome-scale shRNA resource for transgenic RNAi in Drosophila. Nat. Methods 8 , 405-407.

Orngreen, M.C., Vissing, J., Laforet, P., 2015. No effect of bezafibrate in patients with CPTII and VLCAD deficiencies. J. Inherit. Metab. Dis. 38, 373-374.

Pritchard, D.K., Schubiger, G., 1996. Activation of transcription in Drosophila embryos is a gradual process mediated by the nucleocytoplasmic ratio. Genes Dev. 10, $1131-1142$.

Puigserver, P., Spiegelman, B.M., 2003. Peroxisome proliferator-activated receptorgamma coactivator 1 alpha (PGC-1 alpha): transcriptional coactivator and metabolic regulator. Endocr. Rev. 24, 78-90.

Quiros, P.M., Goyal, A., Jha, P., Auwerx, J., 2017. Analysis of mtDNA/nDNA ratio in mice. Curr. Protoc. Mouse Biol. 7, 47-54.

Reeve, S., Carhan, A., Dee, C.T., Moffat, K.G., 2007. Slowmo is required for Drosophila germline proliferation. Genesis. 45, 66-75.

Rørth, P., 1998. Gal4 in the Drosophila female germline. Mech. Dev. 78, 113-118.

Schroder, C., Tautz, D., Seifert, E., Jäckle, H., 1988. Differential regulation of the two transcripts from the Drosophila gap segmentation gene hunchback. EMBO J. 7 , 2881-2887.

Spiegelman, B.M., 2007. Transcriptional control of energy homeostasis through the PGC1 coactivators. Novartis Found. Symp. 286, 3-6.

Steward, R., 1989. Relocalization of the dorsal protein from the cytoplasm to the nucleus correlates with its function. Cell. 59, 1179-1188.

Tiefenböck, S.K., Baltzer, C., Egli, N.A., Frei, C., 2010. The Drosophila PGC-1 homologue Spargel coordinates mitochondrial activity to insulin signalling. EMBO J. 29, 171-183.

Tourmente, S., Lecher, P., Degroote, F., Renaud, M., 1990. Mitochondrial development during Drosophila oogenesis: distribution, density and in situ RNA hybridizations. Biol. Cell. 68, 119-127.

Tracey Jr., W.D., Ning, X., Klingler, M., Kramer, S.G., Gergen, J.P., 2000. Quantitative analysis of gene function in the Drosophila embryo. Genetics. 154, 273-284.

Vercauteren, K., Gleyzer, N., Scarpulla, R.C., 2009. Short hairpin RNA-mediated silencing of PRC (PGC-1-related coactivator) results in a severe respiratory chain deficiency associated with the proliferation of aberrant mitochondria. J. Biol. Chem. 284, 2307-2319.

Wei, P., Guo, J., Xue, W., Zhao, Y., Yang, J., Wang, J., 2018. RNF34 modulates the mitochondrial biogenesis and exercise capacity in muscle and lipid metabolism through ubiquitination of PGC-1 in Drosophila. Acta Biochim. Biophys. Sin. 50, 1038-1046.

Wheeler, J.C., VanderZwan, C., Xu, X., Swantek, D., Tracey, W.D., Gergen, J.P., 2002. Distinct in vivo requirements for establishment versus maintenance of transcriptional repression. Nat. Genet. 32, 206-210. 